\title{
Unconventional neurotransmitters, neurodegeneration and neuroprotection
}

\author{
M. Leonelli, A.S. Torrão and L.R.G. Britto \\ Departamento de Fisiologia e Biofísica, Instituto de Ciências Biomédicas, Universidade de São Paulo, \\ São Paulo, SP, Brasil \\ Correspondence to: L.R.G. Britto, Departamento de Fisiologia e Biofísica, ICB, USP, Av. Prof. Lineu \\ Prestes, 1524, 05508-900 São Paulo, SP, Brasil \\ Fax: +55-11-3091-7426. E-mail: Irbritto@usp.br
}

\begin{abstract}
Neurotransmitters are also involved in functions other than conventional signal transfer between nerve cells, such as development, plasticity, neurodegeneration, and neuroprotection. For example, there is a considerable amount of data indicating developmental roles for the glutamatergic, cholinergic, dopaminergic, GABA-ergic, and ATP/adenosine systems. In this review, we discuss the existing literature on these "new" functions of neurotransmitters in relation to some unconventional neurotransmitters, such as the endocannabinoids and nitric oxide. Data indicating both transcriptional and post-transcriptional modulation of endocannabinoid and nitrinergic systems after neural lesions are discussed in relation to the non-conventional roles of these neurotransmitters. Knowledge of the roles of neurotransmitters in brain functions other than information transfer is critical for a more complete understanding of the functional organization of the brain and to provide more opportunities for the development of therapeutical tools aimed at minimizing neuronal death.
\end{abstract}

Key words: Endocannabinoids; Cannabinoid receptors; Vanilloid receptors; Nitric oxide

Presented at the IV Miguel R. Covian Symposium, Ribeirão Preto, SP, Brazil, May 23-25, 2008.

Research supported by FAPESP (\#07/52947-0 and \#08/53856-1) and CNPq (\#302350/2007-1, \#473352/2007-9). M. Leonelli was supported by a pre-doctoral fellowship from FAPESP (\#05/57127-6).

Received October 2, 2008. Accepted January 29, 2009

\section{The endocannabinoid system}

Endocannabinoids are defined as endogenous ligands of the cannabinoid receptors CB1 and CB2 (1). The basal levels of endocannabinoids are similar in different areas of the brain, including the brainstem, striatum, hippocampus, neocortex, diencephalon, and cerebellum. However, provocative data indicate that there is no direct correlation between the concentration of the endocannabinoids and the expression of the CB1 receptor, which suggests that endocannabinoids could also be involved in other neurotransmitter systems. Actually, it is also known that these compounds can directly modulate several other receptors, such as the serotonergic $5-\mathrm{HT}_{2}$ and $5-\mathrm{HT}_{3}$ receptors, the glutamatergic $\mathrm{N}$-methyl-D-aspartate (NMDA) receptor, and the transient receptor potential vanilloid type 1 (TRPV1) (2).
The first endocannabinoid to be isolated was $\mathrm{N}$-arachidonoylethanolamine, or anandamide, an amide of arachidonic acid with ethanolamine. In 1995, 2-arachidonoylglycerol (2-AG) was discovered, an ester of arachidonic acid with glycerol, which is the most abundant cannabinoid ligand in the central nervous system. Since then, several other lipids, all derived from arachidonic acid, have been shown to modulate the cannabinoid receptors, such as 2arachidonoylglycerol ether (noladin ether), O-arachidonoylethanolamine (virodhamina), and $\mathrm{N}$-arachidonoyldopamine (3).

Both anandamide and 2-AG are synthesized on demand under neuronal stimulation. Anandamide is produced by the enzymes $\mathrm{N}$-acyl-phosphatidylethanolaminehydrolyzing-phospholipase $\mathrm{D}$ and is degraded by fatty acid amide hydrolase. 2-AG is synthesized by diacylglycerol 
lipase and thus degraded by the monoacylglycerol lipases. As hydrophobic compounds, they can reach the extracellular fluid, including synaptic terminals, where they can be taken up by neurons through the anandamide membrane transporter (AMT) (1). It is interesting that 2-AG has been found frequently in brain preparations at much higher concentrations than anandamide. 2-AG seems to occur both in the final steps of the biosynthetic pathway of certain lipids, as well as in the initial steps of the production of other lipids, and thus may participate in cell metabolism rather than solely as a neuromodulator molecule $(3,4)$.

The regulation of the synthesis of these compounds has not been completely elucidated. As an example, the stimulation of NMDA receptors in neuronal cultures from rat cortex yielded a 3 -fold rise in 2-AG production, but no change was observed in anandamide levels. However, simultaneous activation of the NMDA and $\alpha-7$ acetylcholine receptors increased the anandamide levels 5-fold, whereas nicotinic stimulation alone did not cause any changes in the endocannabinoid levels (5). In addition, recent findings have shown that endocannabinoid production is linked to the rise of intracellular calcium elicited by protein $\mathrm{G}_{\mathrm{q} / 11}$-coupled receptor activation (6). Thus, it is clear that the molecular mechanisms that underlie the synthesis of anadamide and 2-AG are distinct and are probably dependent upon the other neuromodulators that share the same synaptic terminal.

\section{Cannabinoid receptors}

Cannabinoid receptors are coupled to $\mathrm{G}_{\mathrm{i} / 0}$ protein that is sensitive to the psychoactive compound delta-9-tetrahydrocannabinol from Cannabis sativa (7). The CB1 receptor was cloned in the early 1990's, and since it is found mainly in neurons, it is known as the neuronal cannabinoid receptor (8). The CB2 receptor was first detected in hematopoietic cells and in the spleen (9) and has been recently shown to be expressed in neuronal and glial cells in the central nervous system (10).

Activation of the CB1 receptor has been extensively implicated in the control of synapse function. Binding of endogenous ligands to CB1 receptors leads to inhibition of adenylate cyclase, thus reducing AMPc levels. This has been implicated in inhibition of pre-synaptic calcium currents and in the activation of potassium rectifying channels, therefore inhibiting the release of several neuromodulators such as glutamate, dopamine, acetylcholine, and norepinephrine $(1,7)$.

Several other intracellular pathways such as mitogenactivated protein-kinase and $\mathrm{STAT}_{3}$ have been shown to be associated with neuronal maturation, axon outgrowth and cellular division. Moreover, the CB1 receptor has also been implicated in the activation of JNK, WAVE and PAK, which have been implicated in the enhancement of actin production and polymerization. In turn, this can lead to neuronal growth (11).

The endocannabinoid system has also been implicated in neuroprotection in neuronal damage models both in vivo and in vitro (12). These studies have shown increased levels of endocannabinoids and upregulation of CB1 receptors in rats and mice, which could represent a protective response. In addition, a receptor reduction is observed in some neurodegenerative diseases, such as Huntington's disease (13).

We reported that unilateral retinal lesions produced an increase in the immunoreactivity for CB1 in the neuropil of superficial layers of the deafferented avian optic tectum and an increased CB1 expression as determined by immunoblotting. Unlike what was observed for protein levels, unilateral retinal ablation did not produce any detectable changes of CB1 mRNA levels (14). We also observed that retinal lesions do not seem to generate cell death by apoptosis in the optic tectum, as evaluated by the TUNEL method (in situ DNA fragmentation staining). The CB1 increase was accompanied by a microtubule-associated protein-2 increase.

This study suggests that, as observed for the nitrinergic system, CB1 expression in visual structures of the chick brain is negatively regulated by the retinal innervation. Since we did not observe cell death in the tectum in this lesion model, the up-regulation of CB1 could indicate the participation of the cannabinoid system in plasticity processes. Alternatively, the up-regulation of $\mathrm{CB} 1$ could have some neuroprotective role as shown in other studies, which have demonstrated increased levels of endocannabinoids and upregulation of $\mathrm{CB} 1$ receptors in brain injury models in rats and mice $(15,16)$. In addition, our results suggest a postsynaptic location of these receptors in tectal layers of the chick brain, despite the fact that several studies have indicated mainly a presynaptic localization for $\mathrm{CB} 1$ receptors as modulators of neurotransmission (3). The CB1 receptors in the tectum could, however, be located on axon terminals of tectal afferents other than those of retinal origin. This issue clearly deserves further investigation.

\section{Vanilloid receptor 1}

The vanilloid receptors belong to the transient receptor potential (TRP) channel, a large family of cation channels mostly permeable to calcium and magnesium. Based on protein homology, the TRP family can be subdivided into seven subfamilies: the vanilloid family (TRPV), the canoni- 
cal family, the melastatin family, the polycystin family, the mucolipin family, the ankyrin family, and the NOMPC family (17).

TRPV is composed of six members (TRPV1-6). TRPV1 was the first channel to be identified in the vanilloid subfamily (18). Given its sensitivity to capsaicin, the pungent compound extracted from the hot chili pepper, the TRPV1 receptor was known as the capsaicin receptor prior to its cloning and sequencing. TRPV1 is also sensitive to endocannabinoids such as anandamide and $\mathrm{N}$-arachidonoyldopamine, and 12(S)-hydroperoxyeicosatetraenoic acid(12(S)-HPETE), which have been also designated as endovanilloids (19). TRPV1 calcium currents can also be elicited by protons and by temperatures above $43^{\circ} \mathrm{C}(19)$.

Activation of TRPV1 has been implicated in several physiological and pathological conditions, such as body temperature control, sensitivity to acids in the gastrointestinal mucosa, modulation of vascular tonus, neuropathic pain, and inflammatory hyperalgesia, among others (19). The TRPV1 receptor is distributed throughout the whole body in different tissues, including a large number of areas in the central nervous system (20).

Early studies with capsaicin showed that this compound can cause degeneration of primary sensory neurons (21) and in an appreciable number of areas of the central nervous system (22). The cell loss elicited by capsaicin has been related to excess inward calcium currents (23).

One should point out the protective roles of CB1 receptors and some other pro-degenerative roles of TRPV1 in inflammatory processes in the central nervous system. However, in addition to the apparent protective role of CB1 receptors, CB1 agonists trigger the release of 12(S)-HPETE in rat mesencephalic cultures, thus activating calcium influx through TRPV1 receptors and causing neuronal death due to mitochondrial damage (24). This single example shows that conclusive statements cannot be made about the real points of interaction between the two systems. Additional studies are required to identify the mechanisms involved in the receptor signaling, metabolic control of synthesis and degradation of the endocannabinoids/ vanilloids.

\section{Signaling in the nitric oxide system}

Nitric oxide (NO) is a freely diffusing gas that can also be found as a free radical (NO in its molecular form), which is involved in diverse physiological processes. In the central nervous system, NO is involved in functions that range from cognition, synaptic plasticity, sleep, neurosecretion, and appetite to temperature homeostasis (25).

Classically, the synthesis of NO depends upon the nitric oxide synthase (NOS) enzymes. All cytosolic NOS isoforms have L-arginine and oxygen as substrates and $\mathrm{NADPH}$ as an electron donor to form $\mathrm{NO}$, thus leading to the production of $\mathrm{NO}$, citrulline and water (26). Three cytosolic NOS isoforms have been identified so far. Two of them are constitutively expressed and are calcium-dependent, and their nomenclature was created based on the cells in which they are predominating found: the neuronal isoform (nNOS, or type 1, NOS-1), and the endothelial isoform (eNOS, or type 3, NOS-3). The inducible isoform (iNOS, or type II, NOS-2) is calcium-independent and its expression was found to be inducible in different tissues. Indeed, it was found that neurons can also express eNOS and that iNOS expression can be induced in neuronal cultures by cytokines. Astrocytes can also express eNOS and, when activated by inflammatory mediators, may express iNOS (27).

In addition to the traditional cytoplasmic NO production sites, NO synthesis has also been reported to occur in mitochondria (28). Several reports indicate that there is a specific mitochondrial isoform of NOS, the mitochondrial NOS. However, recent findings indicate that the release of $\mathrm{NO}$ in the mitochondria of mouse brain, liver and heart is independent of calcium and of L-arginine and is not abolished in the presence of classical NOS inhibitors, such as L-NAME and L-NMMA. In addition, NO production in mitochondria is dependent on the integrity of this organelle and on the interaction between complexes I and II and the ubiquinone complex. There are several possible sources of NO in the mitochondria, such as NO donors like nitrosothiols (29), the conversion of nitrates into NO, and the conversion of organic nitrates to NO reactive species as the other possible sources. In addition, it has been shown that $\mathrm{NO}$ can be also produced by nitrate dismutation or reduction by changes in the $\mathrm{pH}$ of the solution (30). The real existence of mitochondrial NOS is still unclear and this topic is under intense debate even one decade after its first description.

After its production, NO can bind to soluble guanylate cyclase, which in turn converts GMP into cGMP. The cGMP signaling pathway includes the activation of threonine/serine kinase $\mathrm{G}$ (PKG), the activation of sodium and potassium cyclic nucleotide-gated channels, and the activation of several phosphodiesterases, which in turn can modulate PKG action (31).

\section{Nitric oxide in degenerative processes}

NO can generate neuronal death in two basic different ways $(32,33)$. The first mode in which $\mathrm{NO}$ was implicated in neuronal death is by its overproduction. Under these cir- 
cumstances, the presence of reactive oxygen species is needed for the formation of the more reactive nitrogen species. The reaction of $\mathrm{NO}$ with superoxide $\left(\mathrm{O}_{2}^{-}\right)$can yield peroxynitrite (ONOO-), which is extremely reactive and has been implicated in lipid, protein and DNA damage. Peroxynitrate can apparently form hydroxyl radicals $\left(\mathrm{OH}^{\circ}\right)$, which represent very aggressive oxidant substances, and nitrite $\left(\mathrm{NO}_{2}{ }^{\circ}\right)$, or it can also react with $\mathrm{CO}_{2}$, thus forming carbonate anion $\left(\mathrm{CO}_{3}{ }^{-}\right)$, another oxidant. $\mathrm{NO}$ can also react with oxygen and produce $\mathrm{NO}_{2}{ }^{\circ}$, which in turn reacts with other $\mathrm{NO}$ molecules to form dinitrogen trioxide $\left(\mathrm{N}_{2} \mathrm{O}_{3}\right)$. $\mathrm{NO}_{2}$ is able to oxidize or nitrate several molecules. One of them is tyrosine or tyrosine residues in proteins, generating 3-nitrotyrosine (32). On the other hand, $\mathrm{N}_{2} \mathrm{O}_{3}$ can nitrosylate (or nitrosate) amines and thiols (S-nitrosylation) such as glutathione, glyceraldehyde-3 phosphate dehydrogenase, and the NR1 and NR2 subunits of the NMDA receptor, among others (34).

In addition, under acidic conditions such as those found during hypoxia, peroxynitrate can form peroxynitrous acid $(\mathrm{ONOOH})$, which can react in two different ways. One of them is by forming nitrate $\left(\mathrm{NO}_{3}{ }^{-}\right)$, which is not a strong oxidant. The other pathway includes the formation of nitrogen dioxide $\left(\mathrm{NO}_{2}\right)$ and possibly $\mathrm{OH}^{\prime}$, two potent oxidant molecules that can lead to fatty acid oxidation and nitration of amino acids (35).

Excessive NO production was also correlated with neuronal death caused by cessation of mitochondrial respiration (33). NO was found to block in a reversible fashion the mitochondrial cytochrome oxidase, by competing with oxygen. In addition, NO, nitrosothiols, and peroxynitrate were implicated in the reversible inactivation of complexes I and II in mitochondria (36).

These effects of NO on mitochondrial respiration have been implicated in neuronal death caused by the impairment of sodium-potassium-ATPase, neuronal depolarization and consequent glutamate excitotoxicity (33). Coculture of neuronal cells with LPS/IFN- $\gamma$-activated astrocytes and microglial cells (which can produce high levels of NO through iNOS expression) led to neuronal death, which was prevented by co-incubation with an NMDA receptor antagonist (37). In other studies, nNOS blocking prevented cytochrome $\mathrm{C}$ impairment in rat cerebellar granule cell cultures exposed to hypoxia, thus indicating that nNOS activity could be synergistic with hypoxia, leading to neuronal death. This result agrees with that of another study in which cortical neurons of nNOS knock-out mice showed improved resistance to hypoxia and hypoglycemia when compared to wild-type mice. This was also obtained with wild-type mice exposed to NMDA blockers (38).

Several studies have shown up-regulation of $\mathrm{NO}$ and
nNOS observed in the brain after ischemia or in other neuropathological conditions such as Alzheimer's disease (32). Within the context of lesions and degeneration processes related to changes in regulation of nNOS expression, it is important to mention that several studies have been conducted using the vertebrate visual system as a model. However, these studies have reported conflicting results. For example, some studies have shown that eye enucleation generated a down-regulation of nNOS in the superior colliculi and lateral geniculate nuclei of rats (39) and in the optic tectum of the developing chick (40). In contrast, an increased expression of NADPH-diaphorase, a nonspecific NOS marker, was observed after unilateral optic nerve transection in visual centers of the rat (41).

We have shown that unilateral retinal lesions in 4- to 15-day-old chicks (Gallus gallus) produced a marked increase of nNOS immunoreactivity in the neuropil of the superficial layers and in perikarya of the deep layers in the contralateral (deafferented) optic tectum, and increased expression of the enzyme detected by immunoblotting (42). Moreover, we observed a higher production of NO in deafferented tecta using the $\mathrm{NO}$ imaging based on the diaminofluorescein technique. We also observed in this study that retinal lesions caused a marked decrease in the number of neuronal nuclei stained for the nuclear protein Zenk, the product of the early response gene zenk, and in the immunoreactivity of the $160-\mathrm{kD}$ neurofilament.

Taken together, these results suggest that NOS expression in visual structures of the chick brain is regulated negatively by the retinal innervation, which could be an indication of the participation of NO in plasticity processes triggered by deafferentation. Despite the conflicting results reported by different research groups, retinal activity seems to exert an important influence on the expression of NOS in visual structures and these differences could reflect distinct species and developmental stages. It is noteworthy that the experiments cited above with the chick were repeated both in the adult rat and the adult mouse with essentially the same results: up-regulation of nNOS and a greater production of NO. Furthermore, it was also shown that the up-regulation was dependent on a transcriptional mechanism in the visual thalamus, but not in the superior colliculus, pointing to differential regulation mechanisms (43).

\section{Nitric oxide and neuroprotection}

Notwithstanding its potential harmful effects, NO has been shown to actually have beneficial effects in neuronal biology (44). Two pathways that were reported to be activated by the activation of NO-soluble guanylyl cyclase- 
cGMP and the further activation of PKG are AKT (protein kinase $B$ ) and the transcription factor CREB. It has been demonstrated that these pathways are involved in neuronal protection mediated by neurotrophic factors.

S-nitrosylation is also another mechanism by which $\mathrm{NO}$ can modulate proteins that could be involved in degenerative neuronal processes. It was observed that $\mathrm{NO}$ can nitrosylate the NR1 and NR2 subunits of the NMDA receptor (34), thus blocking excitotoxicity mediated by this receptor. S-nitrosylation was also suggested as a mechanism by which NO can modulate caspase activation, thus blocking apoptosis.

NO has also been implicated in the induction of heme oxygenase 1 in rat microglia, in astrocytes and in the hippocampus (45). The expression of this protein increases the biliverdin content of cells, which can be reduced by biliverdin reductase to bilirubin, a potent antioxidant and anti-nitrosative compound that has been suggested to have a role in NO-induced neuroprotection.

\section{Cross-talk between endocannabinoids and nitric oxide}

The relationship between the endocannabinoid and nitrinergic systems has been extensively reported in cell biology studies. This includes distinctive interactions at the level of gene expression and protein function, which are achieved by direct or indirect interaction between molecules of the two systems.

It has been reported that endocannabinoids are released by neurons after brain injury, playing a role in neuronal protection. This was found in models of traumatic brain injury, post-NMDA and kainate excitotoxicity, in focal brain ischemia, and in diabetic retinopathy and macular degeneration $(2,7,12)$.

One example is that, through alpha- $\mathrm{G}_{i}$ activation, $\mathrm{CB} 1$ can modulate calcium inward current and thus modulate constitutive NOS activation. This is particularly important in the mediation of NMDA excitotoxicity. CB1 receptor activation inhibited depolarization of cerebellar granule cells, thus attenuating cellular calcium influx and blocking nNOS activity (46). In mouse cerebral cortex, CB1 activation blocked NO production and reduced cell death (47). In the same study, the investigators found that nNOS activity was higher in cortices of CB1 knockout mice than in normal animals. In the retina, it has been observed that excitotoxicity elicited by NMDA activation is partially caused by NOperoxynitrite formation, which was blocked by co-treatment with delta-9-tetrahydroxycannabinol (48).

In contrast, there is evidence that the direct signaling elicited by CB1 activation can lead to NO production. CB1 activation seemed to lead to NO production in several neuronal culture lines and in brain slices (49). Hence, TRPV1 activation in cat dorsal neck muscles increased NADPH-diaphorase activity in the cervical and lumbar spine, thus indicating that activation of peripheral TRPV1 receptors is involved in central plasticity (50). Recently, it was also reported that CB1 activation in N18TG2 neuroblastoma cells resulted in translocation of the $ß 1$ subunit of soluble guanylyl cyclase to the membrane (51). The investigators speculated that the translocation of soluble guanylyl cyclase to the membrane may be a permissive factor, which provides a higher efficiency in signals triggered by NO stimulation of this enzyme.

$\mathrm{NO}$ has also been implicated in the facilitation of anandamide binding to the TRPV1 receptor. Interestingly, the binding of anandamide to TRPV1 receptor occurs in the intracellular domain of this receptor, and it has been shown that the action of anandamide on the TRPV1 receptor depends on self-production by the cells or by rapid transport of extracellular anandamide to the intracellular space (19). This has been attributed to both diffusion through the cellular membrane and to AMT. AMT activity was shown to be affected by several agents, including $\mathrm{NO}$, which can enhance the transport of anandamide through membranes, permitting anandamide access to the intracellular space, where it can activate the TRPV1 receptor (19).

Cannabinoids have been shown to modulate microglia function. Rat cortical microglial cells express CB1 receptors, and their activation leads to inhibition of NO production (52). It was shown that mouse microglial cells produce higher concentrations of 2-AG when activated by high ATP concentrations. 2-AG and other cannabinoids, through activation of CB2 receptors and abnormal-cannabidiolsensitive receptors and subsequent activation of ERK 1/2, also increased microglial cell migration (53). Conversely, it was found that S-nitrothiols such as S-nitrosoglutathione and S-nitrosocysteine can reversible block CB1 receptor activation of a $\mathrm{G}_{\mathrm{i}}$-protein (54). S-nitrosothiols, as discussed previously, can transnitrosilate several proteins at their thiol residues in a reversible manner, thus modulating the function of diverse proteins.

It has been recently reported that activation of the CB1 receptor by ACEA, a CB1 agonist, and other agonists prevented iNOS expression and cell death in rat forebrain cultures deprived of oxygen and glucose, a model that mimics focal ischemia (55). Despite the direct effects of endocannabinoids on CB1 and CB2 receptors, it has been shown that activation of TRPV1 in isolated mouse retinal microglial cells can induce nuclear factor kappa $ß$ translocation (56), which is known to activate iNOS transcription. 
We have been performing several experiments in order to study the relationship between the nitrinergic and the vanilloid systems in the rat retina (Leonelli $\mathrm{M}$, Martins DO, Britto LRG, unpublished data). Double-labeling immunofluorescence experiments were used to determine whether the TRPV1 receptor is expressed in retinal microglial cells. We found that TRPV1 immunoreactivity was present in OX-42-positive microglial cells, with a preferential localization in cellular processes. We then performed LPS intraocular injections $(5 \mu \mathrm{L}, 1 \mu \mathrm{M})$, which are known to induce iNOS expression. In such cells, we found minor TRPV1 labeling. We also tested whether capsaicin, a TRPV1 agonist, could induce iNOS expression. Intravitreal injections of capsaicin were induced by iNOS expression in these cells, albeit lesser in intensity. In the same model, we performed immunoblotting and immunoperoxidase assays to determine whether capsaicin treatment affected protein nitration. We found a higher 3-nitrotyrosine signal in both experiments, which was completely blocked by the concomitant capsazepine treatment. Recently, it was reported that capsaicin lead to iNOS expression in PC12 cells (57). However, this was not blocked by capsazepine, the TRPV1 receptor antagonist. It was also shown that capsaicin also generates superoxide in C6 glioma cells, thus enhancing nitrosative stress and protein nitrosylation (58). Hence, our experiments indicated that retinal microglial cells expresses the TRPV1 receptor and that its direct activation could lead to nitrosative stress, which was blocked by capsazepine.

Recently, it was demonstrated that TRPV1 activation previous to a high pressure-induced ischemic lesion in the rat retina reduced the loss of retinal ganglion cells (59). In

\section{References}

1. Di Marzo V, Deutsch DG. Biochemistry of the endogenous ligands of cannabinoid receptors. Neurobiol Dis 1998; 5: 386-404.

2. Fride E. Endocannabinoids in the central nervous system an overview. Prostaglandins Leukot Essent Fatty Acids 2002; 66: 221-233.

3. Piomelli $D$. The molecular logic of endocannabinoid signalling. Nat Rev Neurosci 2003; 4: 873-884.

4. Piomelli D, Greengard P. Lipoxygenase metabolites of arachidonic acid in neuronal transmembrane signalling. Trends Pharmacol Sci 1990; 11: 367-373.

5. Stella N, Piomelli D. Receptor-dependent formation of endogenous cannabinoids in cortical neurons. Eur J Pharmacol 2001; 425: 189-196.

6. Ohno-Shosaku T, Hashimotodani Y, Maejima T, Kano M. Calcium signaling and synaptic modulation: regulation of endocannabinoid-mediated synaptic modulation by cal- the same study, these investigators indicated that the high pressure-induced ischemic lesion triggered fatty acid amide hydrolase to a higher expression level, thus lowering anandamide concentrations in the retina. Our results indicate that TRPV1 expression can also be detected in retinal blood vessels. Endocannabinoids seem to modulate vascular tonus by acting both on CB1 and TRPV1 receptors. In fact, NO was also released by TRPV1 stimulation in endothelial cells from rat mesenteric bed (60).

On the basis of these considerations, we hypothesize that TRPV1 protection in the ischemic model could be due to a previous activation of TRPV1 receptor (59) and possibly to eNOS activation and vasodilatation, thus preventing ischemia-induced neuronal damage.

\section{Concluding remarks}

The data presented in this review indicate that the cannabinoid, vanilloid and nitrinergic systems are all involved in several aspects of the neural functions, in addition to conventional neurotransmission. More knowledge of the mechanisms involved in their regulation after brain injury, and of their interactions, is necessary to enable the neurosciences to fulfill their ultimate task, which is the generation of therapeutical tools to minimize neurodegeneration.

\section{Acknowledgments}

Thanks are due to Maria Fernanda R. Graciano (USP) and Eduardo R.L. de Oliveira (University of São Paulo) for critically reading the manuscript. cium. Cell Calcium 2005; 38: 369-374.

7. Howlett AC, Breivogel CS, Childers SR, Deadwyler SA, Hampson RE, Porrino LJ. Cannabinoid physiology and pharmacology: 30 years of progress. Neuropharmacology 2004; 47 (Suppl 1): 345-358.

8. Matsuda LA, Lolait SJ, Brownstein MJ, Young AC, Bonner $\mathrm{TI}$. Structure of a cannabinoid receptor and functional expression of the cloned cDNA. Nature 1990; 346: 561-564.

9. Munro S, Thomas KL, Abu-Shaar M. Molecular characterization of a peripheral receptor for cannabinoids. Nature 1993; 365: 61-65.

10. Onaivi ES, Ishiguro H, Gong JP, Patel S, Perchuk A, Meozzi $\mathrm{PA}$, et al. Discovery of the presence and functional expression of cannabinoid CB2 receptors in brain. Ann N Y Acad Sci 2006; 1074: 514-536.

11. He JC, Neves SR, Jordan JD, lyengar R. Role of the Go/i signaling network in the regulation of neurite outgrowth. Can 
J Physiol Pharmacol 2006; 84: 687-694.

12. Grundy RI, Rabuffetti M, Beltramo M. Cannabinoids and neuroprotection. Mol Neurobiol 2001; 24: 29-51.

13. Glass $M$, Dragunow $M$, Faull $R L$. The pattern of neurodegeneration in Huntington's disease: a comparative study of cannabinoid, dopamine, adenosine and $\operatorname{GABA}(\mathrm{A})$ receptor alterations in the human basal ganglia in Huntington's disease. Neuroscience 2000; 97: 505-519.

14. Chaves GP, Nogueira TC, Britto LR, Bordin S, Torrao AS. Retinal removal up-regulates cannabinoid $\mathrm{CB}(1)$ receptors in the chick optic tectum. J Neurosci Res 2008; 86: 16261634.

15. Hansen HH, Schmid PC, Bittigau P, Lastres-Becker I, Berrendero F, Manzanares J, et al. Anandamide, but not 2arachidonoylglycerol, accumulates during in vivo neurodegeneration. J Neurochem 2001; 78: 1415-1427.

16. Romero J, Berrendero F, Perez-Rosado A, Manzanares J, Rojo A, Fernandez-Ruiz JJ, et al. Unilateral 6-hydroxydopamine lesions of nigrostriatal dopaminergic neurons increased CB1 receptor mRNA levels in the caudate-putamen. Life Sci 2000; 66: 485-494.

17. Pedersen SF, Owsianik G, Nilius B. TRP channels: an overview. Cell Calcium 2005; 38: 233-252.

18. Caterina MJ, Schumacher MA, Tominaga M, Rosen TA, Levine JD, Julius $D$. The capsaicin receptor: a heat-activated ion channel in the pain pathway. Nature 1997; 389: 816-824.

19. Di Marzo V, Blumberg PM, Szallasi A. Endovanilloid signaling in pain. Curr Opin Neurobiol 2002; 12: 372-379.

20. Starowicz K, Nigam S, Di Marzo V. Biochemistry and pharmacology of endovanilloids. Pharmacol Ther 2007; 114: 1333.

21. Jancso G, Kiraly E, Jancso-Gabor A. Pharmacologically induced selective degeneration of chemosensitive primary sensory neurones. Nature 1977; 270: 741-743.

22. Ritter S, Dinh TT. Capsaicin-induced neuronal degeneration in the brain and retina of preweanling rats. J Comp Neurol 1990; 296: 447-461.

23. Grant ER, Dubin AE, Zhang SP, Zivin RA, Zhong Z. Simultaneous intracellular calcium and sodium flux imaging in human vanilloid receptor 1 (VR1)-transfected human embryonic kidney cells: a method to resolve ionic dependence of VR1-mediated cell death. J Pharmacol Exp Ther 2002; 300: 9-17.

24. Kim SR, Bok E, Chung YC, Chung ES, Jin BK. Interactions between $\mathrm{CB}(1)$ receptors and TRPV1 channels mediated by 12-HPETE are cytotoxic to mesencephalic dopaminergic neurons. Br J Pharmacol 2008; 155: 253-264.

25. Guix FX, Uribesalgo I, Coma M, Munoz FJ. The physiology and pathophysiology of nitric oxide in the brain. Prog Neurobiol 2005; 76: 126-152.

26. Bredt DS, Snyder SH. Nitric oxide, a novel neuronal messenger. Neuron 1992; 8: 3-11.

27. Alderton WK, Cooper CE, Knowles RG. Nitric oxide synthases: structure, function and inhibition. Biochem $J$ 2001; 357: 593-615.

28. Ghafourifar P, Richter C. Nitric oxide synthase activity in mitochondria. FEBS Lett 1997; 418: 291-296.

29. Steffen M, Sarkela TM, Gybina AA, Steele TW, Trasseth NJ, Kuehl D, et al. Metabolism of S-nitrosoglutathione in intact mitochondria. Biochem J 2001; 356: 395-402.
30. Zweier JL, Samouilov A, Kuppusamy P. Non-enzymatic nitric oxide synthesis in biological systems. Biochim Biophys Acta 1999; 1411: 250-262.

31. Esplugues JV. NO as a signalling molecule in the nervous system. Br J Pharmacol 2002; 135: 1079-1095.

32. Pacher P, Beckman JS, Liaudet L. Nitric oxide and peroxynitrite in health and disease. Physiol Rev 2007; 87: 315-424.

33. Brown GC, Bal-Price A. Inflammatory neurodegeneration mediated by nitric oxide, glutamate, and mitochondria. $\mathrm{Mol}$ Neurobiol 2003; 27: 325-355.

34. Jaffrey SR, Erdjument-Bromage H, Ferris CD, Tempst $P$, Snyder SH. Protein S-nitrosylation: a physiological signal for neuronal nitric oxide. Nat Cell Biol 2001; 3: 193-197.

35. Beckman JS, Beckman TW, Chen J, Marshall PA, Freeman BA. Apparent hydroxyl radical production by peroxynitrite: implications for endothelial injury from nitric oxide and superoxide. Proc Natl Acad Sci U S A 1990; 87: 1620-1624.

36. Brown GC, Borutaite $\mathrm{V}$. Inhibition of mitochondrial respiratory complex I by nitric oxide, peroxynitrite and S-nitrosothiols. Biochim Biophys Acta 2004; 1658: 44-49.

37. Bal-Price A, Brown GC. Inflammatory neurodegeneration mediated by nitric oxide from activated glia-inhibiting neuronal respiration, causing glutamate release and excitotoxicity. J Neurosci 2001; 21: 6480-6491.

38. Dawson VL, Kizushi VM, Huang PL, Snyder SH, Dawson TM. Resistance to neurotoxicity in cortical cultures from neuronal nitric oxide synthase-deficient mice. J Neurosci 1996; 16: 2479-2487.

39. Zhang C, Granstrom L, Wong-Riley MT. Deafferentation leads to a down-regulation of nitric oxide synthase in the rat visual system. Neurosci Lett 1996; 211: 61-64.

40. Williams CV, Nordquist D, McLoon SC. Correlation of nitric oxide synthase expression with changing patterns of axonal projections in the developing visual system. $J$ Neurosci 1994; 14: 1746-1755.

41. Yan XX, Garey LJ, Liang Y, von Bussmann KA, Jen LS. Increased expression of NADPH-diaphorase in visual centres after unilateral optic nerve transection in the rat. $J$ Hirnforsch 1995; 36: 485-488.

42. Torrao AS, Britto LR. Increased expression of nitric oxide synthase in visual structures of the chick brain after retinal removal. J Neurosci Res 2004; 78: 123-131.

43. Chacur M, Matos RJ, Batista SS, Kihara AH, Britto LR. Differential regulation of the neuronal isoform of nitric oxide synthase in the superior colliculus and dorsal lateral geniculate nucleus of the adult rat brain following eye enucleation. Int J Dev Neurosci 2006; 24: 461-468.

44. Calabrese V, Mancuso C, Calvani M, Rizzarelli E, Butterfield DA, Stella AM. Nitric oxide in the central nervous system: neuroprotection versus neurotoxicity. Nat Rev Neurosci 2007; 8: 766-775.

45. Kitamura $\mathrm{Y}$, Furukawa M, Matsuoka $\mathrm{Y}$, Tooyama I, Kimura $\mathrm{H}$, Nomura $\mathrm{Y}$, et al. In vitro and in vivo induction of heme oxygenase-1 in rat glial cells: possible involvement of nitric oxide production from inducible nitric oxide synthase. Glia 1998; 22: 138-148.

46. Hillard CJ, Muthian S, Kearn CS. Effects of CB(1) cannabinoid receptor activation on cerebellar granule cell nitric oxide synthase activity. FEBS Lett 1999; 459: 277-281.

47. Kim SH, Won SJ, Mao XO, Jin K, Greenberg DA. Molecular mechanisms of cannabinoid protection from neuronal exci- 
totoxicity. Mol Pharmacol 2006; 69: 691-696.

48. El-Remessy AB, Khalil IE, Matragoon S, Abou-Mohamed G, Tsai NJ, Roon P, et al. Neuroprotective effect of (-)Delta9tetrahydrocannabinol and cannabidiol in N-methyl-D-aspartate-induced retinal neurotoxicity: involvement of peroxynitrite. Am J Pathol 2003; 163: 1997-2008.

49. Mukhopadhyay S, Shim JY, Assi AA, Norford D, Howlett AC. $\mathrm{CB}(1)$ cannabinoid receptor-G protein association: a possible mechanism for differential signaling. Chem Phys Lipids 2002; 121: 91-109.

50. Pilyavskii Al, Maznychenko AV, Maisky VA, Kostyukov Al, Hellstrom F, Windhorst U. Capsaicin-induced effects on cfos expression and NADPH-diaphorase activity in the feline spinal cord. Eur J Pharmacol 2005; 521: 70-78.

51. Jones JD, Carney ST, Vrana KE, Norford DC, Howlett AC. Cannabinoid receptor-mediated translocation of NO-sensitive guanylyl cyclase and production of cyclic GMP in neuronal cells. Neuropharmacology 2008; 54: 23-30.

52. Waksman Y, Olson JM, Carlisle SJ, Cabral GA. The central cannabinoid receptor (CB1) mediates inhibition of nitric oxide production by rat microglial cells. J Pharmacol Exp Ther 1999; 288: 1357-1366.

53. Walter L, Franklin A, Witting A, Wade C, Xie Y, Kunos G, et al. Nonpsychotropic cannabinoid receptors regulate microglial cell migration. J Neurosci 2003; 23: 1398-1405.

54. Kokkola T, Savinainen JR, Monkkonen KS, Retamal MD, Laitinen JT. S-nitrosothiols modulate G protein-coupled re- ceptor signaling in a reversible and highly receptor-specific manner. BMC Cell Biol 2005; 6: 21.

55. Fernandez-Lopez D, Martinez-Orgado J, Nunez E, Romero J, Lorenzo P, Moro MA, et al. Characterization of the neuroprotective effect of the cannabinoid agonist WIN-55212 in an in vitro model of hypoxic-ischemic brain damage in newborn rats. Pediatr Res 2006; 60: 169-173.

56. Sappington RM, Calkins DJ. Contribution of TRPV1 to microglia-derived IL-6 and NFkappaB translocation with elevated hydrostatic pressure. Invest Ophthalmol Vis Sci 2008; 49: 3004-3017.

57. Qiao S, Li W, Tsubouchi R, Murakami K, Yoshino M. Role of vanilloid receptors in the capsaicin-mediated induction of iNOS in PC12 cells. Neurochem Res 2004; 29: 687-693.

58. Qiao S, Li W, Tsubouchi R, Haneda M, Murakami K, Yoshino M. Involvement of peroxynitrite in capsaicin-induced apoptosis of C6 glioma cells. Neurosci Res 2005; 51: 175-183.

59. Nucci C, Gasperi V, Tartaglione R, Cerulli A, Terrinoni A, Bari M, et al. Involvement of the endocannabinoid system in retinal damage after high intraocular pressure-induced ischemia in rats. Invest Ophthalmol Vis Sci 2007; 48: 29973004.

60. Poblete IM, Orliac ML, Briones R, Adler-Graschinsky E, Huidobro-Toro JP. Anandamide elicits an acute release of nitric oxide through endothelial TRPV1 receptor activation in the rat arterial mesenteric bed. J Physiol 2005; 568: 539551. 